\title{
Détection de I'ADN cytosolique par la voie cGAS-STING
}

> La synthase de GMP-AMP cyclique (cGAS) est un senseur ubiquitaire d'ADN cytosolique, bien décrite pour reconnaître les acides nucléiques provenant des pathogènes. En présence d'ADN, elle induit la formation d'un messager cellulaire, le GMP-AMP cyclique (cGAMP), qui se lie à STING, une protéine adaptatrice. L'engagement de STING induit la production de cytokines et d'interférons de type I, jouant un rôle majeur dans l'élimination du pathogène. Récemment, un rôle nouveau du complexe cGAS-STING a émergé dans la réponse anti-tumorale. Cette revue synthétise les connaissances actuelles montrant la capacité de cette voie à détecter l'ADN des cellules malignes, ainsi que son rôle dans le contrôle de la tumorigenèse. <

La réponse immunitaire innée est la première ligne de défense contre les infections. Elle repose sur la reconnaissance de motifs essentiels et spécifiques aux pathogènes, les PAMP (pathogen-associated molecular patterns), par des récepteurs spécialisés de l'immunité innée, les PRR (pattern recognition receptors). Des signaux de danger libérés par les cellules endommagées de l'hôte, appelés DAMP (damage-associated molecular patterns) sont également reconnus par ces PRR. Leur engagement induit une signalisation intracellulaire aboutissant à une réponse inflammatoire permettant de lutter contre le pathogène et de réparer le tissu. L'ADN bactérien, qui est enrichi en îlots $C p G$ non méthylés, est détecté par le récepteur membranaire TLR9 (toll-like receptor 9) [1], le LPS (lipopolysaccharide) des membranes de bactéries à Gram négatif, par le TLR4. Les ARN viraux double brins cytosoliques sont, eux, reconnus par les hélicases de type RIG-I (retinoic acid-inducible gene 1 protein). Chez les eucaryotes, I'ADN est normalement confiné dans le noyau de la cellule ou dans la mitochondrie. Sa présence dans le cytosol est détectée par des senseurs de

Vignette (Photo ๑ Inserm - Charlotte Boix)

\section{De l’immunité innée vers le contrôle de la tumorigenèse}

Romain Chanut, Virginie Petrilli

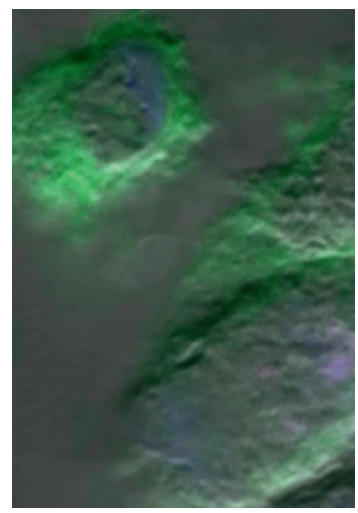

Centre de recherche en cancérologie de Lyon, UM5286 U1052, université de Lyon, centre Léon Bérard, 69008 Lyon, France. virginie.petrilli@lyon.unicancer.fr

I'ADN, tels que AIM2 (absent in melanoma 2), DAI (DNA-dependent activator of IRF) ou IFI16 (interferon gamma-inducible protein 16), dont les spécificités ne sont pas entièrement élucidées [1].

En 2013, le laboratoire de ZJ Chen a identifié la cGAS (cyclic GMPAMP synthase) comme étant un senseur majeur et ubiquitaire de I'ADN cytosolique double brin indépendamment de sa séquence [2]. L'interaction de l'ADN cytosolique avec cGAS entraîne son activation et conduit à la production du second messager cGAMP (cyclic GMP-AMP) à partir d'ATP et de GTP. Ce messager se fixe à son récepteur STING (stimulator of interferon genes) qui est localisé sur la membrane du réticulum endoplasmique (Figure 1). Cette liaison induit son changement de conformation et l'activation des kinases TBKI (TANK-binding kinase 1) et IKK (inhibitor of nuclear factor kappa kinase). Celles-ci phosphorylent respectivement le facteur de transcription IRF3 (interferon regulatory factor 3 ) et $I \kappa B \alpha$, le répresseur de NF- $\kappa B$ (nuclear factor kappa B). La forme phosphorylée d'IRF3 et le facteur de transcription $N F-\kappa B$ ainsi libérés se relocalisent alors dans le noyau où ils induisent l'expression des gènes codant les interférons de type I (IFN-I) et des cytokines inflammatoires. Ces médiateurs contribuent à l'établissement d'une réponse immunitaire contre le pathogène détecté [3]. cGAS ne détecte pas seulement I'ADN d'origine microbienne, mais également celui de l'hôte lorsqu'il se retrouve présent dans le cytosol à la suite d'un stress. Cet ADN est alors considéré comme un DAMP. Cette réponse contre I'ADN de l'hôte peut être à l'origine de maladies auto-immunes, comme le lupus érythémateux disséminé ou le syndrome d'Aicardi-Goutières $[4,56](\rightarrow)$.

$(\rightarrow)$ Voir la Synthèse de P. Lebon et al., $\mathrm{m} / \mathrm{s} \mathrm{n}^{\circ} 3$, mars 2019, page 232 


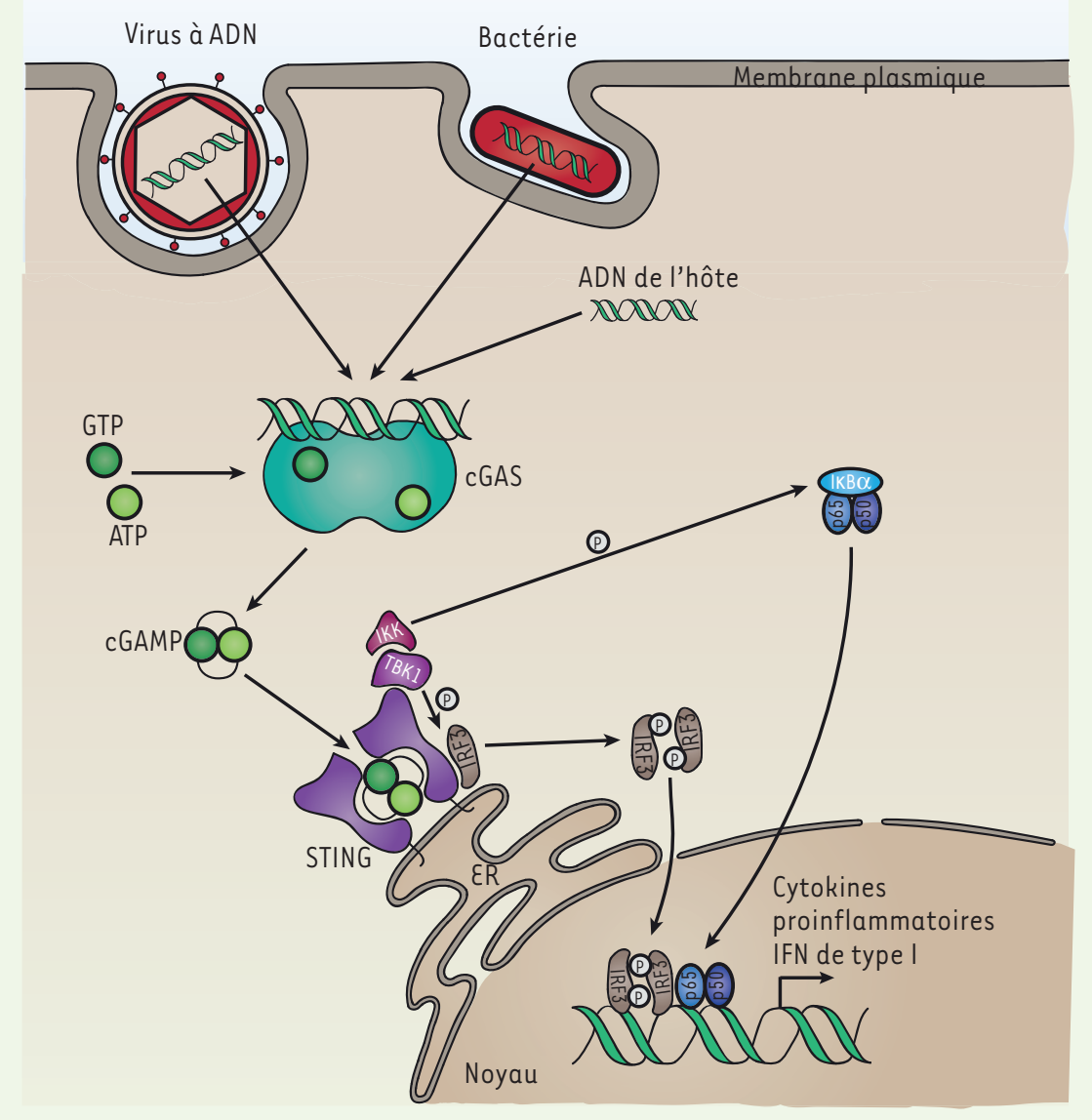

Figure 1. Voie de signalisation cGAS-STING. L'ADN cytoplasmique double brin active cGAS, stimulant la production de cGAMP à partir d'ATP et de GTP. cGAMP se lie à STING et induit son changement de conformation, permettant le recrutement de TBKl et IKK. Ces deux kinases phosphorylent respectivement IRF3 et IKB$\alpha$. La forme phosphorylée de $1 \kappa B \alpha$ libère le facteur NF- $\kappa B$ (sous-unités p65 et p50). Cet hétérodimère et P-IRF3 (IRF3 phosphorylé) entrent dans le noyau où ils induisent l'expression des IFN-I et de cytokines en se liant à leurs promoteurs cibles respectifs. TKBI : TANKbinding kinase 1 ; IKK : inhibitor of NF- $K B$ kinase ; IRF3 : interferon regulatory factor $3 ; \mid \kappa B \alpha: N F-\kappa B$ inhibitor alpha; NF- $\kappa B$ : nuclear factor kappa $B$ (est constitué de plusieurs sous-unités dont p65 et p50); IFN-I : interféron de type I; $\varepsilon R$ : réticulum endoplasmique.

ront à cette surveillance, notamment en modifiant les antigènes qu'elles expriment. Lorsqu'elles l'emportent sur le système immunitaire, tout en induisant un microenvironnement fortement immunosuppresseur, on parle de l'étape d'échappement, qui correspond à la progression tumorale et à

L'expression ubiquitaire de cGAS lui confère la capacité de surveiller de multiples événements dans l'organisme. Depuis la découverte de ce PRR, ce domaine de recherche est en ébullition. Les nombreuses études récentes n'ont pas seulement contribué à mieux comprendre son rôle dans la détection des pathogènes, mais elles ont révélé de nouvelles fonctions pour ce PRR dans la réponse anti-tumorale. Dans cette revue, nous nous intéresserons aux travaux montrant les nouvelles fonctions de la voie cGAS-STING dans la détection des stress génotoxiques et dans le contrôle de la progression tumorale.

\section{La voie cGAS-STING, une barrière anti-tumorale}

\section{L'immunosurveillance des cellules cancéreuses}

Il existe une relation étroite entre les cellules cancéreuses et le système immunitaire. Un modèle propose que ce dernier détermine le destin des cellules cancéreuses au cours de 3 étapes : «les $3 \varepsilon$ \& [5]. La première étape est dite d'élimination, car le système immunitaire reconnaît et élimine les cellules aux premiers stades de la tumorigenèse. Lors de l'étape qui la suit, l'étape dite d'équilibre, le système immunitaire jugule les cellules tumorales qui, en réponse, s'adaptent continuellement à cet environnement immunitaire. Certaines cellules cancéreuses échappe- l'apparition des métastases [5].

Les IFN-I jouent un rôle primordial dans l'activation de l'immunité anti-tumorale. Ces IFN agissent sur les CPA (cellules présentatrices d'antigènes $[\mathrm{Ag}]$ ) en stimulant la présentation des $\mathrm{Ag}$ en association avec le complexe majeur d'histocompatibilité de classe I ( $\mathrm{CMH}-\mathrm{I})$. Des Ag produits par des cellules tumorales, qui ont été phagocytées par les CPA, sont ainsi présentés, de façon dite croisée, aux lymphocytes T CD8 cytotoxiques (LTC). La reconnaissance des Ag par les LTC induit leur activation et conduit à la sécrétion de cytokines dont le TNF- $\alpha$ (tumor necrosis alpha), de perforines et de granzyme $B$, qui agiront de concert pour détruire la cellule cible tumorale. La capture d'ADN tumoral par des APC, comme les DC (cellules dendritiques), activent la voie cGAS-STING et induit la production d'IFN-I et de cytokines, comme l'interleukine 12 (IL-12), ainsi que l'expression de molécules de costimulation, telles que CD40, CD80 (ou B7.1) et CD86 (ou B7.2), et de facteurs qui sont nécessaires à l'activation des lymphocytes $T$ CD8 [6-9] (Figure 2). La stimulation des DC par des 


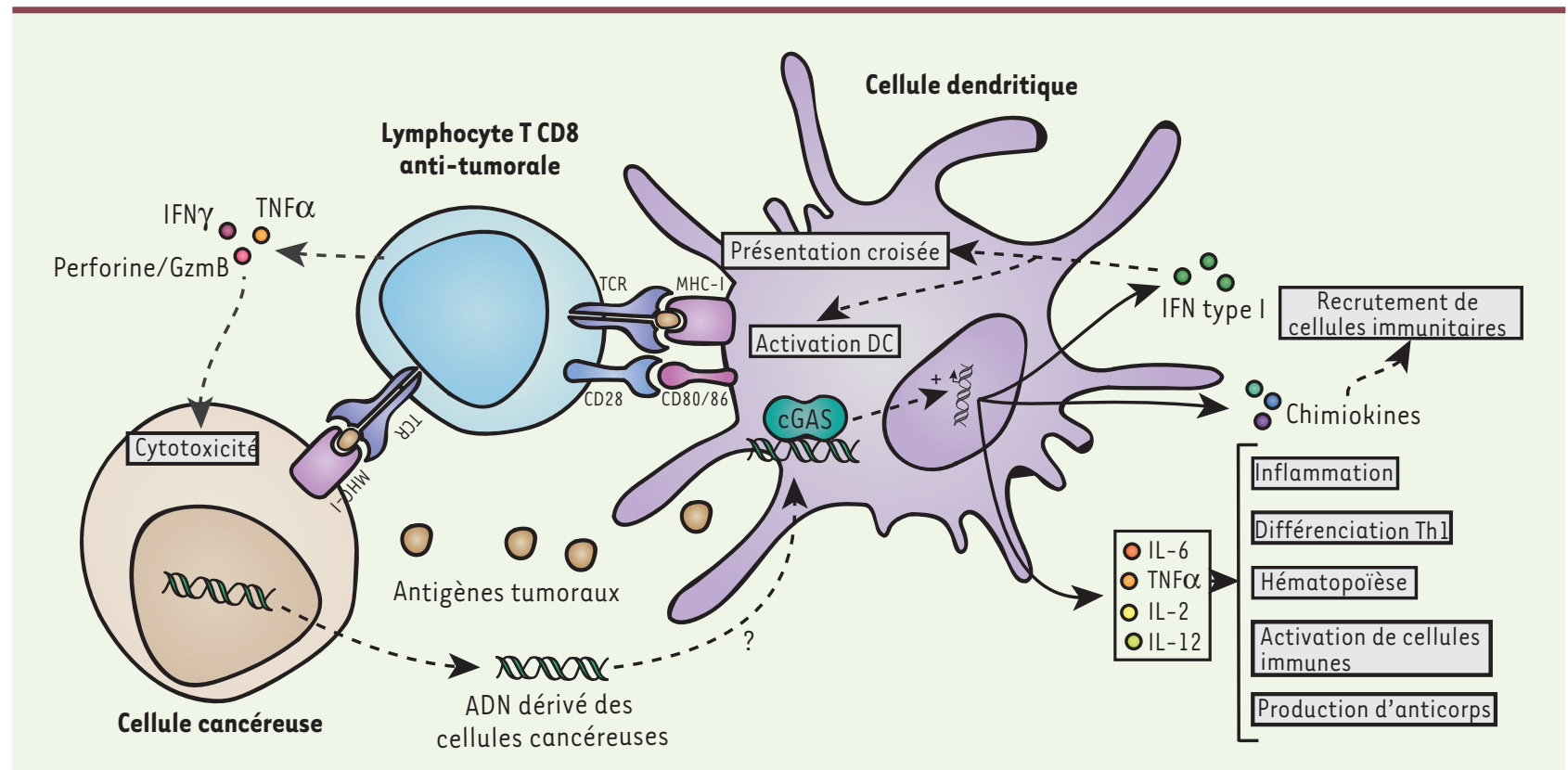

Figure 2. cGAS et immunité anti-tumorale. L'activation de cGAS dans les cellules immunitaires conduit à la production de cytokines proinflammatoires et d'IFN-I indispensables à la mise en place de réponses immunitaires spécifiques telles que la présentation croisée des antigènes tumoraux, l'inflammation, la différenciation des lymphocytes Thl, l'activation des cellules immunitaires et la production d'anticorps ainsi qu'à une stimulation de l'hématopoï̀se. Des chimiokines sont également produites et permettent le recrutement de cellules immunitaires. DC : cellule dendritique ; GzmB : granzyme B ; MHC-I : complexe majeur d'histocompatibilité de type I ; TCR : T cell receptor.

cellules tumorales irradiées induit ainsi la présentation croisée d’Ag tumoraux et la stimulation de lymphocytes T CD8 naïfs. Cet effet est perdu chez des souris qui n'expriment pas STING, suggérant que l'absence de STING diminue l'immunité anti-tumorale [7, 10$]$.

À l'inverse, le traitement de souris par cGAMP, ou un ligand synthétique de STING, stimule l'immunité anti-tumorale et réduit la croissance de la tumeur $[7,10,11]$ en induisant une immunité adaptative mémoire [12]. Le traitement par cGAMP, combiné à la stimulation du TLR4 par du LPS, permet également d'activer NLRP3 (NOD-like receptor family, pyrin domain-containing 3), un PRR cytosolique senseur de danger [13]. Cela conduit à la formation de l'inflammasome, complexe contrôlant l'activation de la caspase 1, à l'origine de la pyroptose, une forme de mort cellulaire, et à la production d'IL-1 $\beta$ et d'IL-18, qui contribuent à la mise en place d'une immunité anti-tumorale.

L'immunothérapie est une stratégie thérapeutique anti-tumorale qui vise à bloquer les points de contrôle inhibiteurs (comme PD-1 [programmed death 1] ou CTLA4 [cytotoxic-T-lymphocyte-antigen 4 protein]) exprimés par les cellules immunitaires, afin de restaurer leur capacité à détruire les cellules cancéreuses. Dans un modèle murin de mélanome, l'inactivation génétique de la voie cGAS-STING diminue drastiquement l'efficacité de l'immunothérapie, se traduisant par un recrutement inefficace des LTC dans les tumeurs $[9,10,14]$. cGAS et STING apparaissent donc essentiels au développement d'une immunité anti-tumorale efficace.

Mais quelle est la source d'ADN endogène capable d'activer cGAS dans un contexte tumoral?
Au sein du microenvironnement tumoral, les cellules cancéreuses sont exposées à des stress hypoxiques, mécaniques et métaboliques qui peuvent conduire à leur mort. La mort des cellules cancéreuses peut également avoir pour origine les thérapies appliquées (chimiothérapie, radiothérapie). Les cellules mourantes libèrent alors des composants qui sont reconnus par les PRR comme des signaux de danger (les DAMP), en particulier leur ADN. L'ADN tumoral est capté par des $A P C$, comme les DC, dans lesquelles il induit l'activation de cGAS. Dans les contextes infectieux, cGAS peut être activé par un ADN microbien qui peut être contenu dans des vésicules extracellulaires produites par les cellules infectées $[15,16]$. Les cellules cancéreuses libèrent également ce type de vésicules. Les exosomes, qui sont de petites vésicules membranaires, contiennent en effet des acides nucléiques et des protéines issus des cellules. Il est donc possible que cGAS puisse être activé par ce biais [17], mais les mécanismes moléculaires par lesquels I'ADN tumoral capté par les DC atteint le cytosol de ces cellules restent à définir.

\section{Activation intrinsèque de cGAS-STING par les dommages à l'ADN}

L'instabilité génétique est un élément important du développement tumoral [18]. Une cellule normale 
possède différents programmes impliqués dans le maintien de l'intégrité de son génome: les voies de réparation de l'ADN, l'arrêt du cycle cellulaire et, si les dommages sont trop importants, l'induction de l'apoptose ou de la sénescence. Lorsque les gènes impliqués dans ces processus de sauvegarde sont mutés ou inactivés, les cellules prolifèrent malgré la présence de dommages sur leur ADN, entraînant alors plus de dommages et l'accumulation de mutations, et, in fine, la transformation de la cellule. Les agents génotoxiques, comme les irradiations ou les chimiothérapies, induisent la production d'IFN-I et de cytokines proinflammatoires, suggérant un rôle de la voie cGAS dans la détection des dommages à I'ADN [19, 20].

Les senseurs d'ADN, comme l'est cGAS, constituent donc un mécanisme permettant de détecter l'ADN endommagé et d'empêcher l'initiation tumorale. La radiothérapie et la chimiothérapie, qui reposent sur l'induction de dommage à l'ADN dans le but de tuer les cellules cancéreuses, activent la voie cGAS [21, 22] et plusieurs études ont montré que des dysfonctionnements dans les voies de réparation de l'ADN résultaient en l'activation de cGAS (Figure 3). Ainsi, la perte de fonction, par mutation ou par invalidation, d'ATM (ataxia telangiectasia mutated), un régulateur clé de la signalisation contrôlant les voies de réparation de l'ADN, induit la production d'IFN-I via la voie cGAS-STING, in vitro et in vivo $[23,24]$. La perte de Rad51 et de RPA (replication protein $A$ ), deux protéines majeures de la réparation d'ADN, conduit également à l'activation de cGAS, in vivo et in vitro dans des modèles cellulaires de cancers ovariens et mammaires [25]. En situation physiologique, lorsque l'ADN est répliqué ou réparé, de l'ADN simple brin est généré et est lié à ces protéines. Leur délétion induit donc l'accumulation de cet ADN et son passage vers le cytosol. STING peut également détecter les dommages, indépendamment de cGAS, en formant un complexe avec p53 et IFI16 lorsque la voie ATM est activée [26].

De nombreuses mutations affectant les voies de réparation de I'ADN conduisent à une accumulation cytoplasmique d'ADN nucléaire dans plusieurs modèles de cellules cancéreuses. Cet ADN provient de l'activité de facteurs impliqués dans la résection de l'ADN, comme BLM (Bloom helicase) ou EX01 (exonuclease 1), dont la sous-expression réduit l'accumulation dans le cytoplasme [27-29]. Lors de dommages à l'ADN, le clivage par l'endonucléase MUS81 au niveau des fourches de réplication combiné à l'activation de la PARPI (poly[ADP-ribose] polymerase 1 , une enzyme impliquée dans la détection des cassures d'ADN) sont responsables de l'accumulation d'ADN nucléaire au niveau du cytoplasme et de l'activation de STING [27]. L'ADN mitochondrial endommagé en réponse à un stress métabolique peut également fuiter vers le cytoplasme $[30,31]$. Les cellules cancéreuses ont une forte demande métabolique. Elles sont également plus sujettes à un stress métabolique. Une fuite d'ADN mitochondrial pourrait donc contribuer à l'activation de cGAS, bien qu'aucune étude n'ait adressé cette question à ce jour.

En situation physiologique, l'ADN qui atteint le cytosol est généralement immédiatement dégradé par des nucléases comme TREXI (three prime repair exonuclease 1). Néanmoins, étant donné le haut niveau de mutations des cellules en cours de transformation ou déjà cancéreuses, la quantité d'ADN cytosolique peut surpasser la capacité de TREXI à le dégrader. II n'est également pas exclu que TREXI puisse être inactivée par des mutations, prévenant ainsi la dégradation de I'ADN dans le cytoplasme $[32,33]$. Des modifications chimiques de I'ADN (comme le 8-hydroxyguanosine ${ }^{1}$ ) induites par des carcinogènes peuvent aussi le protéger de la dégradation par les nucléases cytoplasmiques, exacerbant ainsi son accumulation dans le cytoplasme et donc, potentiellement, l'activation de cGAS [33]. Dans différents modèles tumoraux, de fortes doses d'irradiations peuvent induire l'expression de TREXI, celle-ci inhibant les effets systémiques de la radiothérapie combinée à l'immunothérapie, en empêchant la production d'IFN-I [34].

Les télomères constituent les extrémités des chromosomes. Ils se raccourcissent au cours des divisions cellulaires conduisant les cellules vers la sénescence. Les cellules cancéreuses échappent à ce mécanisme de raccourcissement par des mécanismes de recombinaison homologue. Elles maintiennent ainsi leurs télomères, ce qui leur permet une prolifération illimitée et l'échappement à la sénescence. Dans ces cellules, l'accumulation de séquences, les ECTR (extrachromosomal telomere repeats), nécessaires pour le maintien des télomères a été décrite. Ces séquences s'accumulent, notamment dans le cytoplasme. Elles sont également capables d'activer cGAS-STING, soulignant, encore une fois, le rôle de cette voie dans la détection des cellules cancéreuses [35].

Malgré toutes ces études montrant une accumulation d'ADN au niveau du cytoplasme, les mécanismes par lesquels I'ADN nucléaire traverse l'enveloppe nucléaire sont peu connus. cGAS rejoindrait I'ADN nucléaire lors de la mitose, mais le rôle de cette localisation particulière reste encore inconnu [36].

\section{Détection d'anomalies chromosomiques}

Les dommages à I'ADN peuvent conduire à la formation de structures chromosomiques aberrantes au cours de la division cellulaire. Les cassures double brin, associées à une réparation défectueuse, peuvent en effet conduire à une mauvaise séparation des chromosomes lors de la mitose. Lorsque les cellules sortent de la mitose, ces chromosomes, ou fragments d'ADN mal séparés, sont alors entourés d'une enveloppe nucléaire formant un micronoyau qui est une caractéristique fréquemment observée dans les cellules cancéreuses [37]. Deux études récentes ont montré que cGAS était capable de reconnaître I'ADN présent dans ces micronoyaux $[11,36]$ (Figure 3). Dans des modèles

\footnotetext{
${ }^{1}$ Ribonucléoside résultant de l'oxydation de la guanosine.
} 


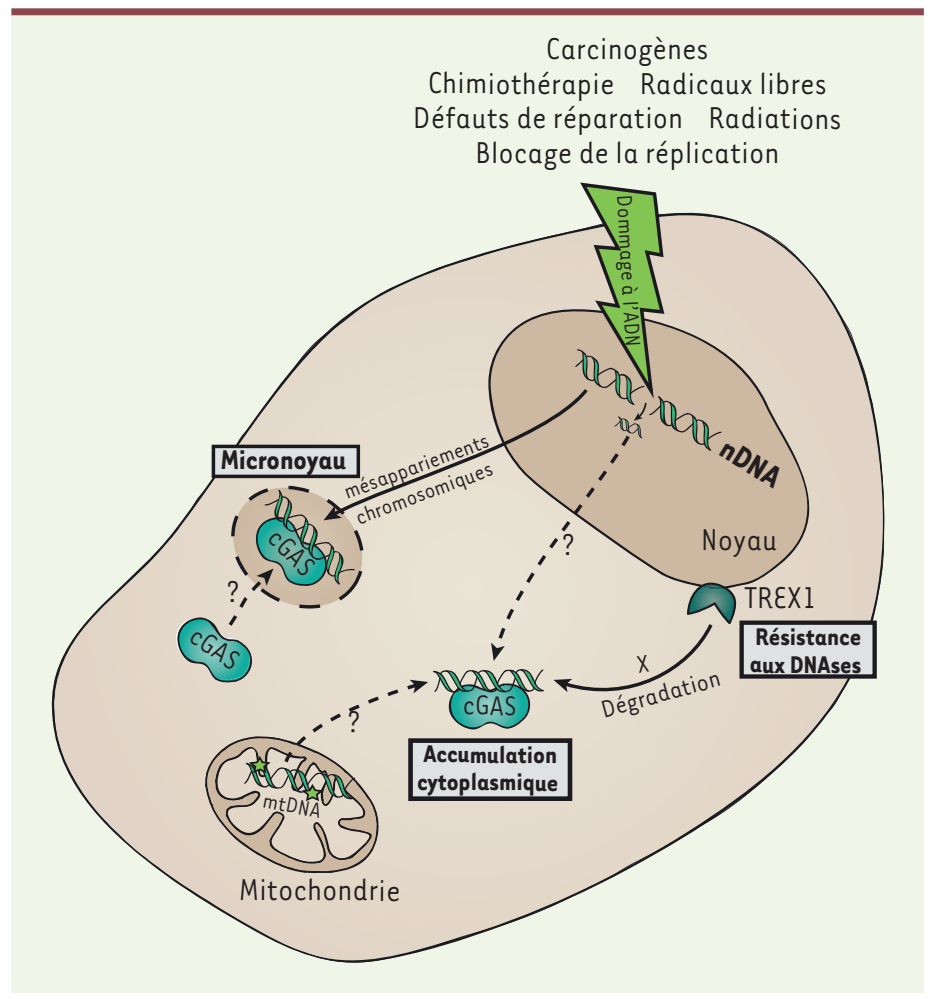

Figure 3. Reconnaissance d'ADN endogène dans les cellules cancéreuses par cGAS. Les cellules cancéreuses accumulent une instabilité génomique du fait de réparation inefficace et de dommages récurrents de leur ADN. Ceci peut conduire à l'accumulation d'ADN dans le cytoplasme et activer cGAS. Les dommages peuvent également engendrer une mauvaise séparation de l'ADN durant la division cellulaire résultant en la formation de micronoyaux, qui sont également reconnus par cGAS. Certains dommages à I'ADN, en induisant des modifications sur les bases, confèrent une résistance aux DNAses et favorisent l'accumulation cytoplasmique d'ADN. nDNA: ADN nucléaire; mtDNA : ADN mitochondrial.

cellulaires présentant un fort taux de micronoyaux (les fibroblastes embryonnaires murins [MEF] déficients pour la RNase H2 et pour le suppresseur de tumeur p53, et les cellules épithéliales d'ostéosarcome) cGAS se localise dans ces structures nucléaires [36]. cGAS est également retrouvé associé aux micronoyaux induits par irradiations de cellules épithéliales mammaires [14, 38]. Dans les deux cas, cette localisation de cGAS est à l'origine de l'activation de STING et de la mise en place d'une réponse inflammatoire [36]. L'association de cGAS avec les micronoyaux nécessite la progression du cycle cellulaire $[14,36]$ et la rupture mécanique de l'enveloppe nucléaire, qui précède souvent des réarrangements chromosomiques massifs amenant à l'oncogenèse [36]. Le mécanisme permettant l'entrée de cGAS dans les micronoyaux reste néanmoins mal défini.

\section{Activation des programmes de sauvegarde cellulaire}

La sénescence est un processus agissant comme une barrière à la tumorigenèse. Elle promeut un état antiprolifératif en arrêtant de façon irréversible le cycle cellulaire. cGAS joue un rôle impor- tant dans l'induction et le maintien du processus de sénescence. Dans des modèles de fibroblastes murins et humains, cGAS se localise à proximité de fragments chromatiniens situés à la frontière entre noyau et cytosol. Des MEF déficients pour le gène codant cGAS (cGAS $\left.{ }^{-/}\right)$s'immortalisent spontanément et plus rapidement que les MEF qui l'expriment. Les MEF $\mathrm{CGAS}^{-1-}$ échappent en fait à la sénescence [39-41]. Ces cellules défectives expriment faiblement les marqueurs de sénescence, tels que la $\beta$-galactosidase ou la protéine $\mathrm{pl6}$. Et la sécrétion du SASP (senescence-associated secretory phenotype), un profil de sécrétion caractéristique des cellules senescentes constitué, entre autres, d'IL- $1 \alpha$, d'IL-6, de TNF $\alpha$ et de chimiokines, comme CXCL10 ( $C-X-C$ motif chemokine ligand 10) stimulant le recrutement de cellules immunitaires, y est altérée (Figure 4). cGAS est également essentiel pour induire la sénescence en réponse à des stress génotoxiques ou à l'activation oncogénique [39-41]. cGAS semble donc fortement impliqué dans l'induction de la sénescence in vitro. II contribuerait aussi à la sénescence paracrine en contrôlant le SASP. In vivo, des souris déficientes pour STING ou cGAS et exposées à des inducteurs de sénescence (par irradiations ou surexpression hépatique de l'oncogène NRas) montrent une diminution des marqueurs de sénescence dans le foie et les poumons [39, 40]. Les hépatocytes surexprimant I'oncogène NRas persistent plus longtemps dans le foie des souris $\mathrm{CGAS}^{-1-}$, suggérant un défaut d'élimination de ces cellules par le système immunitaire, ce qui peut conduire à la génération de tumeurs [39, 40]. La sénescence dépendant de la voie cGAS-STING se révèle donc être une importante barrière à la tumorigénèse in vivo.

L'étude de STING dans d'autres modèles cellulaires a révélé son effet anti-prolifératif indépendamment de son rôle sur la stimulation des IFN de type I [42, 43]. Dans les lymphocytes $T$, son activation par un agoniste induit l'apoptose des cellules par une voie impliquant les facteurs IRF-3 et p53; elle serait liée à une stimulation prolongée de cette voie [43]. Cette réponse apoptotique est maintenue dans les lymphomes $T$, ouvrant des perspectives thérapeutiques intéressantes pour les agonistes de STING.

Un autre mécanisme de sauvegarde impliquant STING a également été mis en évidence. L'activation de STING, à la suite de dommages à l'ADN, induit l'expression de ligands à la surface des cellules, reconnus par les récepteurs NKG2D (natural killer group 2D) [44, 45] exprimés par les cellules NK, ce qui induit leur mort par des mécanismes similaires à ceux utilisés par les LTC. 


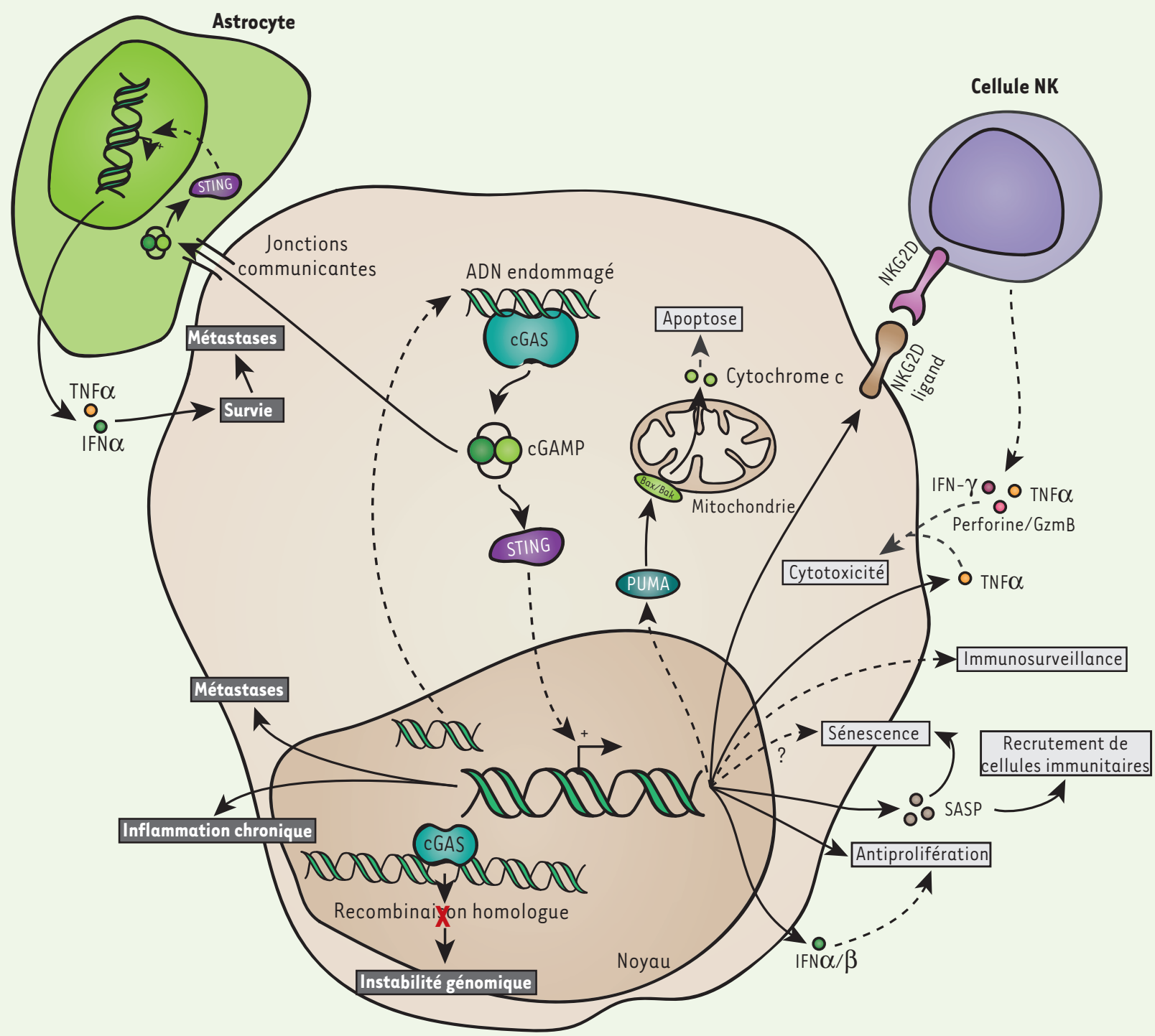

Figure 4. Dualité des réponses tumorales reposant sur cGAS-STING. L'activation de cGAS dans les cellules en cours de transformation induit différentes réponses antitumorales comme la mise en place d'une réponse immunitaire anti-tumorale. La voie conduit également à l'expression de NKG2D qui induit la reconnaissance des cellules cancéreuses par les cellules NK (natural killer) causant une mort cellulaire ciblée des cellules cancéreuses. La production de SASP par cGAS induit un état de sénescence. L'activation de STING induit l'apoptose. L'activation continue de cGAS peut induire une tumorigenèse liée à une inflammation chronique. Cette voie peut également contribuer à une augmentation de l'instabilité génomique en inhibant les voies de réponses aux dommages à l'ADN. Elle peut enfin favoriser l'apparition de métastases. GzmB : granzyme B ; NKG2D : natural killer group 2D; SASP : senescence-associated secretory phenotype ; PUMA : Bcl-2 binding component 3.

\section{cGAS-STING, une épée à double tranchant ?}

Bien que les fonctions anti-tumorales de la voie cGAS soient de mieux en mieux documentées, des études récentes montrent que cette voie pourrait en réalité être une épée à double tranchant, en jouant un rôle pro-tumoral par une action directe sur la tumorigenèse.
Le DMBA (7,12-diméthylbenzène[a]anthracène) est un carcinogène connu pour induire, chez la souris, des tumeurs cutanées dépendant d'une inflammation chronique. Dans des cultures in vitro de fibroblastes murins ou de kératinocytes humains, le DMBA induit une accumulation d'ADN dans le cytoplasme des cellules 
et une forte production de cytokines inflammatoires et d'IFN-I. Cette induction est strictement dépendante de cGAS et de STING, suggérant un rôle de cette voie dans la carcinogenèse cutanée. In vivo, le traitement de souris déficientes pour STING, ou de souris chimériques ayant reçu des cellules de moelle osseuse provenant de souris $S T I N G^{-/-}$par le DMBA n'induit pas d'inflammation cutanée et de tumeurs, soulignant le rôle de cGAS/STING dans la tumorigenèse induite par l'inflammation chez la souris [46].

cGAS pourrait également jouer un rôle dans l'initiation de la tumorigénèse en inhibant les voies de réparations de I'ADN, augmentant ainsi l'instabilité génomique. Liu et al. ont en effet montré une translocation nucléaire de cGAS au niveau des cassures double brin. cGAS interagit avec PARPl, empêchant la recombinaison homologue $(\mathrm{RH})$ qui en dépend. In vitro et in vivo, la déplétion de cGAS favorise ainsi la réparation de l'ADN par $\mathrm{RH}$ et réduit la croissance de la masse tumorale pulmonaire, in vivo [47]. En plus de ce rôle potentiel dans l'initiation tumorale, cGAS pourrait être impliqué dans la progression tumorale en contribuant à la formation de métastases. L'instabilité chromosomique, notamment la formation de micronoyaux et l'accumulation d'ADN cytoplasmique, engage la voie STING et active la voie non canonique de NF-KB impliquée dans l'invasion et la formation de métastases cérébrales, mammaires et ganglionnaires [48]. cGAS peut également avoir des effets paracrines néfastes. Par le biais des jonctions communicantes, les cellules cancéreuses mammaires ou pulmonaires délivrent le cGAMP qu'elles produisent aux astrocytes où il activera STING, à l'origine de la sécrétion de facteurs de survie pour les cellules métastatiques, favorisant ainsi l'établissement de métastases cérébrales [49].

\section{Conclusion et perspectives}

Les senseurs d'ADN et leur rôle dans le cancer représentent un champ de recherche émergeant, en particulier à la suite de la découverte récente du rôle de cGAS en tant que détecteur ubiquitaire d'ADN cytosolique. La voie impliquant cGAS est bien décrite pour la détection de pathogènes et l'induction d'une immunité antimicrobienne. Nous donnons ici une nouvelle vision du rôle de cGAS comme détecteur d'ADN dans la surveillance des tumeurs et l'induction d'une réponse anti-tumorale par la mise en place d'une réponse immunitaire, l'induction de la mort cellulaire et l'inhibition de la prolifération. La voie cGAS-STING façonne le microenvironnement tumoral et alerte l'organisme des dommages qui s'accumulent à l'intérieur des cellules.

Considérant son rôle anti-tumoral, la voie cGAS-STING pourrait être exploitée en thérapie contre le cancer. Des agonistes de cGAS ou de STING ont montré des effets thérapeutiques dans différents modèles murins tumoraux, tels que des mélanomes, des tumeurs du côlon, de la prostate, du sein, et des fibrosarcomes, en recrutant des lymphocytes T CD8 antitumoraux. De nouvelles possibilités d'utilisation des agonistes de cette voie s'ouvrent donc, comme adjuvants de la radiothérapie, de la chimiothérapie et de l'immunothérapie [10, 50]. Des approches innovantes, telles que des particules virales contenant cGAMP, offrent également des perspectives thérapeutiques intéressantes afin d'induire la voie de façon ciblée dans les tumeurs [51].

Il n'est cependant pas surprenant que les cellules cancéreuses échappent à cette voie de signalisation, ce qui peut rendre cette approche thérapeutique difficile à appliquer. Chez l'homme, dans des adénocarcinomes du côlon, et dans des modèles de mélanomes, l'expression de cGAS ou STING est diminuée par l'hyperméthylation de leurs promoteurs. La voie de signalisation est également perturbée en raison de la séquestration de STING au niveau du réticulum endoplasmique $[52,53]$. De même, les tumeurs fortement hypoxiques répriment cGAS par l'induction de microARN régulateurs [54]. Pour les cancers viroinduits, la protéine $\varepsilon 6$ du papillomavirus humain et la protéine $\varepsilon l A$ des adénovirus inhibent cGAS, bloquant ainsi la réponse antivirale et promouvant l'oncogenèse virale [55]. Néanmoins, l'invalidation de la voie cGAS-STING dans les cellules cancéreuses offre une opportunité de cibler ces cellules via des thérapies utilisant des virus oncolytiques [53].

Dans cette revue, nous avons décrit les activités antitumorales de cGAS. La biologie apparaît cependant beaucoup plus complexe. En effet, cette voie se révèle être de plus en plus une épée à double tranchant, les preuves que cGAS pourrait avoir des activités protumorales s'accumulant. Ces fonctions pro-tumorales doivent donc être prises en compte lors de la conception de protocoles thérapeutiques visant à activer la voie cGAS-STING. Une meilleure connaissance dans la manière dont cGAS détecte I'ADN endommagé des tumeurs, ainsi que les réponses tumorales passant par son activation, sont nécessaires afin d'envisager de telles thérapies. $\diamond$

\section{SUMMARY}

Cytosolic DNA sensing by the cGAS-STING pathway in cancer

Cyclic GMP-AMP synthase (cGAS) is a universal cytosolic DNA sensor that detects nucleic acids of pathogens. Upon DNA sensing, cGAS triggers the formation of the second intracellular messenger, the cyclic GMP-AMP (cGAMP), which activates the adaptor STING. STING engagement induces the secretion of cytokines and type I interferons that contribute to pathogen clearance. However, there is emerging evidence that cGAS is activated by self DNA in cancer cells and in antigenpresenting cells to trigger an antitumoral response. In this review, we will highlight the current understanding of self DNA sensing by cGAS in the context of cancer. $\diamond$ 


\section{REMERCIEMENTS}

Les auteurs remercient Marie-Cécile Michallet et Baptiste Guey pour la relecture du manuscrit.

\section{LIENS D’INTÉRÊT}

Les auteurs déclarent n'avoir aucun lien d'intérêt concernant les données publiées dans cet article.

\section{RÉFÉRENCES}

1. Pandey S, Kawai T, Akira S. Microbial sensing by Toll-like receptors and intracellular nucleic acid sensors. Cold Spring Harb Perspect Biol $2015 ; 7$ : a016246.

2. Sun L, Wu J, Du F, et al. Cyclic GMP-AMP synthase is a cytosolic DNA sensor that activates the type I interferon pathway. Science $2013 ; 339: 786-91$

3. Chen Q, Sun L, Chen ZJ. Regulation and function of the cGAS-STING pathway of cytosolic DNA sensing. Nat Immunol 2016; 17 : 1142-9.

4. Crow YJ, Manel N. Aicardi Goutières syndrome and the type I interferonopathies. Nat Rev Immunol $2015 ; 15: 429-40$

5. Schreiber RD, Old LJ, Smyth MJ. Cancer Immunoediting: Integrating immunity's roles in cance suppression and promotion. Science $2011 ; 331: 1565-70$.

6. Xu MM, Pu Y, Han D, et al. Dendritic cells but not macrophages sense tumor mitochondrial DNA for cross-priming through signal regulatory protein signaling. Immunity $2017 ; 47: 363-73$.e5.

7. Deng L, Liang H, Xu M, et al. STING-dependent cytosolic DNA sensing promotes radiation-induced type i interferon-dependent antitumor immunity in immunogenic tumors. Immunity $2014 ; 41$ : $843-52$.

8. Klarquist J, Hennies CM, Lehn MA, et al. STING-mediated DNA sensing promotes antitumor and autoimmune responses to dying cells. I Immunol 2014 ; 193 : 6124-34.

9. Woo S-R, Fuertes MB, Corrales L, et al. STING-dependent cytosolic DNA sensing mediates innate immune recognition of immunogenic tumors. Immunity $2014 ; 41: 830-42$.

10. Wang $\mathrm{H}, \mathrm{Hu} \mathrm{S}$, Chen $\mathrm{X}$, et al. cGAS is essential for the antitumor effect of immune checkpoint blockade. Proc Natl Acad Sci USA 2017 ; 114 : 1637-42.

11. Li T, Cheng H, Yuan H, et al. Antitumor activity of cGAMP via stimulation of cGAS-cGAMP-STINGIRF3 mediated innate immune response. Sci Rep 2016; 6 : 19049.

12. Corrales L, Glickman LH, McWhirter SM, et al. Direct activation of STING in the tumor microenvironment leads to potent and systemic tumor regression and immunity. Cell Rep 2015; $11: 1018-30$.

13. Swanson KV, Junkins RD, Kurkjian CJ, et al. A noncanonical function of cGAMP in inflammasome priming and activation. J Exp Med 2017 ; 214 : 3611-26.

14. Harding SM, Benci JL, Irianto J, et al. Mitotic progression following DNA damage enables pattern recognition within micronuclei. Nature 2017 ; $548: 466-70$.

15. Torralba D, Baixauli F, Villarroya-Beltri C, et al. Priming of dendritic cells by DNA-containing extracellular vesicles from activated T cells through antigen-driven contacts. Nat Commun 2018 $9: 2658$.

16. Sisquella $X$, Ofir-Birin $Y$, Pimentel MA, et al. Malaria parasite DNA-harbouring vesicles activate cytosolic immune sensors. Nat Commun $2017 ; 8$ : 1985.

17. Whiteside TL. Tumor-derived exosomes and their role in cancer progression. Adv Clin Chem 2016 ; $74: 103-41$.

18. Hanahan D, Weinberg RA. Hallmarks of cancer: The next generation. Cell $2011 ; 144: 646-74$

19. Burnette $B C$, Liang $H$, Lee $Y$, et al. The efficacy of radiotherapy relies upon induction of type I interferon-dependent innate and adaptive immunity. Cancer Res 2011; 71 : 2488-96.

20. Pauli W, Valkó $\varepsilon$. Zur Elektrochemie der Kolloide. Kolloid-Zeitschrift $1934 ; 66$ : 312-6

21. Pépin G, Nejad C, Thomas BJ, et al. Activation of cGAS-dependent antiviral responses by DNA intercalating agents. Nucleic Acids Res 2017 ; 45 : 198-205.

22. Wilkins $A C$, Patin $\varepsilon C$, Harrington KJ, et al. The immunological consequences of radiation induced DNA damage. J Pathol $2019 ; 247: 606-14$.

23. Härtlova A, Erttmann SF, Raffi FA, et al. DNA damage primes the type I interferon system via the cytosolic DNA sensor STING to promote anti-microbial innate immunity. Immunity $2015 ; 42$ 332-43.

24. Quek H, Luff J, Cheung K, et al. Rats with a missense mutation in Atm display neuroinflammation and neurodegeneration subsequent to accumulation of cytosolic DNA following unrepaired DNA damage. J Leukoc Biol 2017 ; 101 : 927-47.

25. Wolf C, Rapp A, Berndt N, et al. RPA and Rad51 constitute a cell intrinsic mechanism to protect the cytosol from self DNA. Nat Commun $2016 ; 7: 11752$.

26. Dunphy G, Flannery SM, Almine JF, et al. Non-canonical activation of the DNA sensing adaptor STING by ATM and IFIl6 mediates NF-KB signaling after nuclear DNA damage. Mol Cell $2018 ; 71$ : 745-60.e5.

27. Ho SSW, Zhang WYL, Tan NYJ, et al. The DNA structure-specific endonuclease MUS81 mediates DNA sensor STING-dependent host rejection of prostate cancer cells. Immunity $2016 ; 44: 1177-89$.

28. Parkes $\varepsilon \varepsilon$, Walker SM, Taggart LE, et al. Activation of STING-dependent innate immune signaling by S-phase-specific DNA damage in breast cancer.J Natl Cancer Inst 2017 ; 109 : djw199.
29. Erdal $\varepsilon$, Haider S, Rehwinkel J, et al. A prosurvival DNA damage-induced cytoplasmic interferon response is mediated by end resection factors and is limited by Trexl. Genes Dev $2017 ; 31: 353-69$.

30. Carroll $\varepsilon C$, Jin L, Mori A, et al. The vaccine adjuvant chitosan promotes cellular immunity via DNA sensor cGAS-STING-dependent induction of type I interferons. Immunity $2016 ; 44: 597-608$.

31. West AP, Khoury-Hanold W, Staron M, et al. Mitochondrial DNA stress primes the antiviral innate immune response. Nature 2015 ; 520 : 553-7.

32. Wang CJ, Lam W, Bussom $S$, et al. TREXI acts in degrading damaged DNA from drug-treated tumor cells. DNA Repair (Amst) 2009; $8: 1179$

33. Gehrke N, Mertens C, Zillinger T, et al. Oxidative damage of DNA confers resistance to cytosolic nuclease TREXI degradation and potentiates STINGdependent immune sensing. Immunity 2013 ; 39 : 482-95.

34. Vanpouille-Box C, Alard A, Aryankalayil MJ, et al. DNA exonuclease Trex] regulates radiotherapy-induced tumour immunogenicity. Nat Commun $2017 ; 8: 15618$.

35. Chen $Y-A$, Shen $Y-L$, Hsia H-Y, et al. Extrachromosomal telomere repeat DNA is linked to ALT development via cGAS-STING DNA sensing pathway. Nat Struct Mol Biol $2017 ; 24$ : 1124-31.

36. Mackenzie KJ, Carroll P, Martin CA, et al. cGAS surveillance of micronuclei links genome instability to innate immunity. Nature 2017 ; $548: 461-5$.

37. Fenech M, Kirsch-Volders M, Natarajan AT, et al. Molecular mechanisms of micronucleus, nucleoplasmic bridge and nuclear bud formation in mammalian and human cells. Mutagenesis $2011 ; 26: 125-32$.

38. Mackenzie KJ, Carroll P, Lettice L, et al. Ribonuclease $\mathrm{H} 2$ mutations induce a cGAS/STING-dependent innate immune response. EMBO J 2016 ; 35 : 831-44.

39. Glück S, Guey B, Gulen MF, et al. Innate immune sensing of cytosolic chromatin fragments through cGAS promotes senescence. Nat Cell Biol $2017 ; 19: 1061-70$.

40. Dou Z, Ghosh K, Vizioli MG, et al. Cytoplasmic chromatin triggers inflammation in senescence and cancer. Nature 2017 ; 550 : 402-6.

41. Yang $\mathrm{H}$, Wang $\mathrm{H}$, Ren J, et al. cGAS is essential for cellular senescence. Proc Natl Acad Sci USA 2017 ; 114 : E4612-20.

42. Cerboni S, Jeremiah N, Gentili M, et al. Intrinsic antiproliferative activity of the innate sensor STING in T lymphocytes. J Exp Med 2017 ; 214 : 1769-85.

43. Gulen MF, Koch U, Haag SM, et al. Signalling strength determines proapoptotic functions of STING. Nat Commun $2017 ; 8: 427$

44. Gasser $S$, Orsulic $S$, Brown $\varepsilon$, et al. The DNA damage pathway regulates innate immune system ligands of the NKG2D receptor. Nature $2005 ; 436$ 1186-90.

45. Lam AR, Bert N Le, Ho SSW, et al. RAEl ligands for the NKG2D receptor are regulated by STING-dependent DNA sensor pathways in lymphoma. Cancer Res $2014 ; 74: 2193-203$.

46. Ahn J, Xia T, Konno H, et al. Inflammation-driven carcinogenesis is mediated through STING. Nat Commun $2014 ; 5: 5166$.

47. Liu $\mathrm{H}$, Zhang $\mathrm{H}$, Wu $\mathrm{X}$, et al. Nuclear cGAS suppresses DNA repair and promotes tumorigenesis. Nature $2018 ; 563: 131-6$.

48. Bakhoum SF, Ngo B, Laughney AM, et al. Chromosomal instability drives metastasis through a cytosolic DNA response. Nature 2018 ; $553: 467-72$.

49. Chen $\mathrm{Q}$, Boire $\mathrm{A}$, Jin $\mathrm{X}$, et al. Carcinoma-astrocyte gap junctions promote brain metastasis by cGAMP transfer. Nature 2016 ; $533: 493-8$.

50. Baird JR, Friedman D, Cottam B, et al. Radiotherapy combined with nove STING-targeting oligonucleotides results in regression of established tumors. Cancer Res 2016; $76: 50-61$.

51. Gentili M, Kowal J, Tkach M, et al. Transmission of innate immune signaling by packaging of cGAMP in viral particles. Science 2015 ; 349 : 1232-6.

52. Xia T, Konno H, Barber GN. Recurrent loss of STING signaling in melanoma correlates with susceptibility to viral oncolysis. Cancer Res $2016 ; 76$ : 674759 .

53. Xia T, Konno H, Ahn J, et al. Deregulation of STING signaling in colorectal carcinoma constrains DNA damage responses and correlates with tumorigenesis. Cell Rep 2016; $14: 282-97$.

54. Wu MZ, Cheng WC, Chen SF, et al. miR-25/93 mediates hypoxia-induced immunosuppression by repressing cGAS. Nat Cell Biol 2017 ; 19 : 1286-96.

55. Lau L, Gray $\varepsilon \varepsilon$, Brunette RL, et al. DNA tumor virus oncogenes antagonize the cGAS-STING DNA-sensing pathway. Science 2015 ; 350 : 568-71.

56. Lebon P, Crow YJ, Casanova JL, Gresser I. Conséquences pathologiques d'un excès d'interféron in vivo. Comment ne pas sous- estimer des expériences in vivo quand elles peuvent paraître anecdotiques et non conformistes. Med Sci (Paris) $2019 ; 35: 232-5$

\section{TIRÉS À PART}

\section{Petrilli}

